\title{
Quantitative techniques for medical equipment maintenance management
}

Article in European J of Industrial Engineering · January 2017

CITATIONS

0

5 authors, including:

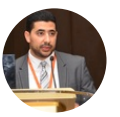

Malek Masmoudi

Université Jean Monnet

30 PUBLICATIONS 37 CITATIONS

SEE PROFILE

Khatrouch Ikram

Université Jean Monnet

13 PUBLICATIONS 2 CITATIONS

SEE PROFILE
READS

93
Ahmad Al Hanbali

University of Twente

43 PUBLICATIONS 450 CITATIONS

SEE PROFILE

Faouzi Masmoudi

University of Sfax

81 PUBLICATIONS 177 CITATIONS

SEE PROFILE 


Quantitative techniques for medical equipment
maintenance management

\title{
Zeineb Ben Houria ${ }^{1,2 *}$, Malek Masmoudi ${ }^{2}$, Ahmad Al Hanbali ${ }^{3}$, Ikram Khatrouch ${ }^{2}$ and Faouzi Masmoudi ${ }^{1}$
}

\author{
${ }^{1}$ University of National School of Engineers of Sfax, Dept. of Mechanical Engineering, \\ LA2MMP, B.P. 1173, 3038 Sfax Sfax, Tunisia \\ ${ }^{2}$ University Saint Etienne, Jean Monnet, Dept. of Maintenance and Industrial \\ Engineering, LASPI, Roanne, France \\ ${ }^{3}$ University of Twente, Industrial Engineering and Business Information Systems, 7500 \\ AE Enschede, the Netherlands \\ E-mail: zeineb.ben.houria@univ-st-etienne.fr \\ E-mail: Malek.Mamsoudi@univ-st-etienne.fr \\ E-mail: a.alhanbali@utwente.nl \\ E-mail: Ikram.Khatrouch@univ-st-etienne.fr \\ E-mail: Faouzi.masmoudi@enis.rnu.tn \\ *Corresponding author
}

\begin{abstract}
The maintenance department in a hospital is responsible for ensuring the safety of medical equipment and their availability while keeping the operation costs minimal. The selection of the best maintenance strategy is a key decision to reduce the equipment downtime, increase the availability, and bring down the maintenance costs. In this paper, we use an integrated approach that includes several tools from the literature, namely, the Analytical Hierarchy Process (AHP), the Technique for Order Preference by Similarity to Ideal Solution (TOPSIS), and the mathematical optimization (especially mixed integer problems MILP) to provide the decision maker of the maintenance department with an entire solution to the problem at hand. These three tools are introduced to 1) determine the criticality of medical equipment based on a multi-criteria analysis, 2) rank the different maintenance strategies based on their (benefits) importance to the hospital and 3) select the optimal maintenance strategy for each device while keeping the total maintenance costs within a predetermined budget. We applied our approach to a case study at the Hospital of "Habib Bourguiba" in Tunisia, and the numerical results show the efficiency of our approach to improve the availability and the reliability of high risk medical devices.
\end{abstract}


Keywords: Multi-criteria Decision-Making; Medical equipment; Maintenance strategies; AHP; TOPSIS; MILP

Biographical notes: Zeineb Ben Houria, is a student of doctor of philosophy degree at National school of Engineers of Sfax at the University of Sfax in Tunisia (Cotutelle with University of Jean-Monnet, Saint Etienne in France). She is working on the optimization of the maintenance of medical devices with a collaborative project with Habib Bouguiba Tunisian Hospital. Her research interests include maintenance optimization models and mathematical modeling. She received her Bachelor of Science degree in industrial engineering from the National School of Engineers of Bizerte, Tunisia.

Malek Masmoudi, is assistant professor in industrial engineering at the University of Jean-Monnet, Saint Etienne in France. He obtained his doctor of philosophy degree in industrial engineering from the University of Toulouse in France.

Ahmad Al Hanbali, is associate professor in industrial engineering and business information systems at the University of Twente, Enschede, the Netherlands. He is a full member of the Beta Research School, the Netherlands; the International Society of Inventory Research; Twente Is Maintenance Excellence; POMS; and Service Logistics Forum. He was a member of many technical program committees of international conferences. He served as a reviewer for several international journals.

Ikram Khatrouch, researcher at the University of Jean-Monnet, Saint Etienne in France. She obtained her doctor of philosophy degree in industrial engineering Science at the University of Sfax-Tunisia. Her research interests include human resource management, hospital logistics and risk management.

Faouzi Masmoudi, is full professor at the National School of Engineers of Sfax in Tunisia and a researcher at the Mechanics Modelling and Production Research Unit (U2MP). He obtained his doctor of philosophy degree in computer-integrated manufacturing (1988) from ENSAM, Paris, France. 
Quantitative techniques for medical equipment maintenance management

\section{Introduction}

The maintenance of medical equipment is crucial for achieving the goal and the objective of health care providers. This is because it mainly contributes in keeping equipment up and running, reducing the rate of failures, keeping the safety requirements, and improving the quality of the healthcare service (Jamshidi et al. 2015). Medical equipment continue to increase in number and complexity to satisfy the increasing demand for health services (e.g., there are more than few thousand equipment in a midsize Tunisian Hospital). In the last few decades, faced to the sophistication of medical equipment and their price that continues to escalate, the complexity and the cost of equipment maintenance have also risen as well (Jamshidi et al. 2014). Usually, the maintenance costs are high due to the inappropriate decision for selecting and fine tuning the correct maintenance strategy. Few studies exist in healthcare area to help the decision maker in selecting the best maintenance strategy for medical equipment (Jamshidi et al. 2014). In this paper, we mainly focus on this important problem.

Maintenance activities are divided into two categories: preventive and corrective (Ebeling 2004). In a corrective maintenance strategy the equipment is run until failure and only when this occurs it receives a repair action on that moment (so no preventive maintenance is done before the equipment failure). This strategy is usually applied for non-critical equipment with an unpredictable failure behavior. However, for equipment suffering from wear-out phenomenon preventive maintenance strategy is typically applied. In preventive maintenance strategy the equipment is preventively maintained based on calendar time, operations time (usage), or state condition. Traditionally, condition based maintenance was done based on inspections done by operators. However, nowadays with the advancement of communication and sensing technology the remote monitoring and diagnosis is becoming possible. The main challenge here is to correctly link the equipment failure to the correct state variable(s) which is typically an equipment (or component) specific. In the context of Tunisian Hospitals, Ltaief et al. (2007) identified three possible strategies: time-based maintenance, condition-based maintenance, and corrective maintenance. In the Time-Based Maintenance (TBM) the equipment is periodically checked according to the calendar time prescribed by the original equipment manufacturer (OEM). This strategy is able to reduce the number of failures and the downtime to an absolute minimum if it is applied 
correctly. Taghipour et al. (2011) reported that with TBM the equipment is usually over maintained due to uncertainty involved in finding the length maintenance interval. Condition-Based Maintenance (CBM) can be better and more cost effective than Time-Based Maintenance (Yang 2003). CBM requires regular assessment of the system condition during its operation. It is carried out in response to significant deterioration in the equipment or a piece of it by monitoring the equipment condition (using direct state variables) or the performance (indirect state variables). This strategy is the most suited for capital intensive equipment with complex and expensive replaceable components. CBM requires a good knowledge of the technical failure mechanisms of the equipment. It involves prognostic and diagnostic analysis and a proper execution of the maintenance planning (Ilangkumaran and Kumanan 2009), (Ding et al. 2014). We note in the Tunisian hospital context the monitoring in CBM is done based on regular inspection by the operators. These operators determine which components are deemed to be in good state and which has to be replaced preventively. Corrective maintenance $(\mathrm{CM})$ is a reactive strategy of unplanned actions due to an equipment failure. This maintenance strategy can be costly due to unplanned downtimes and to inefficient use of the biomedical maintenance staff (Taghipour et al. 2011).

Taghipour et al. (2011) used AHP method to classify the medical equipment according to their criticality. The criticality is computed based on the criteria and the sub-criteria weight. The devices considered to be critical are included in the equipment management program (incl. maintenance) of the hospital. The maintenance strategy of equipment should be done according to the available resources, in terms of budget, human resources, and tools. Recently, Jamshidi et al. (2015) used a fuzzy multi-criteria decision making approach in order to prioritize medical devices based on different expert's opinions and considering uncertainties. They then proposed a maintenance planning diagram to identify the adequate maintenance strategy for each device based on the total intensity score of the multi-criteria analysis and the risk priority index. For a related work but not specifically focusing on the medical equipment maintenance we refer to Ghosh et al. (2010). To the best of our knowledge, there is no procedure in the literature to find the criticality thresholds according to which the maintenance strategy of equipment switches from one strategy to another. This raises a related issue on how to rank the maintenance strategies according to their (benefit) importance to the hospital operations. Our contribution in this paper is threefold: 1 ) we determine the criticality of 
medical equipment based on a multi-criteria analysis, 2) we consider a systematic approach based on TOPSIS procedure to rank and classify the different maintenance strategies, 3) finally, we optimize the criticality thresholds using MILP model in which the budget and the resources capacity constraints are satisfied.

A careful study should be done to select the adequate maintenance strategy in order to reduce the number of failures. Maintenance strategy selection problem is a multi-criteria decision making (MCDM) problem where many criteria have a considerable impact on the decision process. Examples of the criteria are the equipment function, the risk involved, and the equipment's age.

This paper starts with the AHP method to determine the weights of the different criteria in a systematic and consistent way, which are used to find the overall equipment criticality. We find that the function criterion has the highest weight. Moreover, a TOPSIS technique is introduced to help the decision maker to prioritize maintenance strategies used in hospitals. Finally, a MILP model is developed for selecting the optimal strategy for each medical device.

The remainder of this paper is organized as follows: in Section 2, we present the related literature. In Section 3, the proposed approach model for maintenance selection is presented and the stages are explained in details. In Section 4, the experimental results and the data analysis are discussed. Finally, the conclusions of this study and the future research are reported in Section 5.

\section{Literature review}

The major challenge of the biomedical maintenance service is to implement the best maintenance strategy which improves the availability and the efficiency of medical equipment, controls the rate of equipment deterioration, ensures safety and minimizes the total cost (AAMI, 1985). A "risk-based inclusion criteria" approach is proposed by Fennigkoh and Smith (1989) using three main factors: the equipment function, the physical risk on patients, and the maintenance requirements. An equipment management (EM) factor is calculated (representing equipment criticality) as the sum of scores of the three proposed criteria. The devices with an EM above a threshold, e.g., larger than 12, are considered to be relatively critical and thus have to be included in the inspection and the maintenance program. 
Wang and Levenson (2000) proposed a new interpretation of the function criterion called the mission criticality. They define this criterion as the equipment role or importance within the global healthcare organization's mission as opposed to the individual clinical department. Moreover, they introduce the utilization rate of an equipment, i.e., the average percentage of time the equipment is being used. They argue that a low utilization yields not only less urgency for equipment repairs but also less criticality for the equipment. The new calculation of the Equipment Management Rating (EMR) proposed in (Wang and Levenson 2000) is a weighted sum of the different criteria:

$$
\begin{aligned}
& E M R=[(\text { Mission Critical }+2 * \text { maintenance requirements }) * \text { utilization } \\
& \text { rate }]+2 * \text { physical risk }
\end{aligned}
$$

A multi-criteria decision-making (MCDM) model to prioritize the medical equipment according to their criticality is presented in (Taghipour et al. 2011). An Analytical Hierarchy Process (AHP) is used to calculate the weights of criteria and define the criticality score of the medical devices. Moreover, a calculation of the transformed score value (TSV) score is detailed in Taghipour et al. (2011) to establish a guideline for the selection of an appropriate maintenance strategy. Recently, a risk-based maintenance framework for prioritization of medical equipment using fuzzy multicriteria decision making (FMCDM) is proposed by Jamshidi et al. (2015). In this paper, a maintenance strategy classification is proposed through a simple graphic diagram based on the total intensity score of multiple criteria and the risk priority index for each device using fuzzy failure modes and effects analysis (FFMEA). To the best of our knowledge, only these two papers consider the classification of maintenance strategies of medical equipment.

It is surprising to see that the selection of an appropriate maintenance strategy for medical equipment using multi-criteria decision making (MCDM) methods has been rarely used. Despite the fact that there are several papers dealing with the maintenance strategy selection in different industries, as shown in the following. For example, in production, a maintenance decision making model based on two decisions theory topics: utility theory (UT) and multi-attribute utility theory (MAUT) is proposed by Almeida and Bohoris (1995). An application of Analytic Hierarchy Process (AHP) coupled with a sensitivity analysis for selecting the best maintenance strategy for a newly proposed integrated gasification and 
combined cycle plant in an Italian oil refinery is presented in Bevilacqua and Braglia (2000). Five maintenance strategies are considered and the adequate one is selected for each facility of the plant. An integrated model to help taking a maintenance decision using Analytic Hierarchy Process (AHP) and Fuzzy Logic is developed by Labib et al. (1998). In this paper, two steps are described: the first one is the prioritization of machines according to their criticality using AHP model and the second is to select the most appropriate maintenance strategy based on fuzzy logic. The use of AHP method in determining the weights of importance of a pavement maintenance management system is proposed by Ramadan et al. (1999). These weights are then used to classify the maintenance strategies of a road network. A technique for the order preference by similarity to the ideal solution (TOPSIS) as a new multi-criteria decision-making (MCDM) tool is suggested by Hwang and Yoon (1981). This technique is based on a positive and a negative ideal solution, which are determined in respect to the distance of each alternative to the best and the worst performing alternatives respectively. TOPSIS is the one of the best methods to address the rank reversal issue (Ertugrul and Karakasoglu 2007). A the combination of AHP and TOPSIS technique to select the suitable maintenance policy for a textile spinning mill ring frame unit is proposed by Shyjith et al. (2008). Based on the previous literature review the multi-criteria Decision Analysis (MCDA) techniques that can be applied to the medical equipment maintenance are: the AHP and the Analytic Network Process (ANP) to calculate criteria weights based on the experts' judgment, and the Elimination and Choice Expressing Reality (ELECTRE) and TOPSIS technique to classify and rank the maintenance strategies Ivlev et al. (2014).

To conclude this section, we selected AHP method and TOPSIS technique due to their wide applicability in different industries and proven quality in classifying maintenance strategies which include the experts' judgment on both the proposed criteria and the alternative maintenance strategies. Involving expert opinion in the decision process helps in implementing and admitting our proposal in practice. Finally, a MILP model is developed using weights (importance factor) of alternatives obtained on TOPSIS in order to select the best maintenance strategy for each device that maximizes the total importance and satisfies the hospital budget and resources constraints. 


\section{The Proposed model}

For selecting the appropriate maintenance strategy of each device in a hospital, we propose the approach in Figure 1 as a support tool to the decision maker. In the proposed approach, we combine two methods of multi-criteria decision making: AHP and TOPSIS in order to classify the maintenance strategies used in the hospital and MILP for selecting the adequate maintenance strategy to each medical device.

Figure 1 Proposed methodology for selecting maintenance strategy for medical equipment

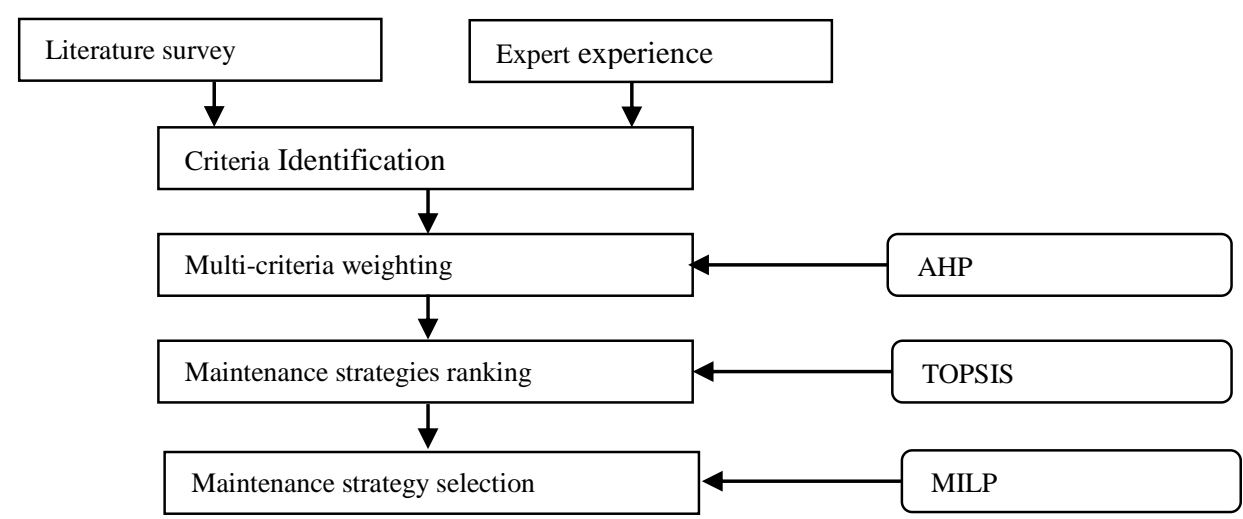

In section 3.1, we explain how to find the weighting factors using AHP method. In section 3.2, we use TOPSIS technique to rank the maintenance strategies according to the equipment criticality. In section 3.3, we determine the criticality thresholds using a MILP model. Since we consider three maintenance strategies we will determine two thresholds $\left(T_{1}\right.$ and $\left.T_{2}\right)$. All equipment with a criticality below $T_{1}$, relative low criticality will have a corrective maintenance strategy. Equipment with criticality above $T_{1}$ will have a either condition based or time based preventive maintenance strategy depending on the criticality (whether it is below or larger than $T_{2}$ ).

\subsection{Calculation the weight of criteria using AHP}

Multi-Criteria Decision Making (MCDM) is a widely used for making preference decisions such as prioritization, evaluation and selection over available alternatives characterized by multiple criteria (Taghipour et al. 2011). 
Analytical Hierarchy Process (AHP) is a flexible multi-Criteria Decision Making tool and can be used for both qualitative and quantitative MCDM problems (Saaty 1980). To highlight the relative importance of a criterion, AHP method uses a simple paired comparison of criteria, which relies on the experts' judgments. AHP method allows to effectively solve a range of practical tasks through the paired comparisons and the hierarchical decomposition principle (Hummel 2001; Linkov et al. 2006). Hence, the AHP method closely reproduces the natural, intuitive and typically human process of defining priorities).

Briefly, the AHP encompasses three mains steps (Hwang and Yoon 1981; Saaty 1994):

1 Establishment of a hierarchy structure: decomposing the complex problem into a hierarchy structure from an overall decision objective to various criteria, sub-criteria, and the lowest level in a descending order.

2 Establishment of comparative judgments: Decision makers compare each criterion (sub-criterion) to all other criteria (sub-criteria) at the same level of the hierarchy using a pairwise comparison matrices introduced to find criteria weights.

3 Evaluation of alternatives: Decision makers evaluate the decision alternatives taking into account the weight of different criteria. The optimal solution is the alternative with the greatest cumulative weight.

\subsubsection{Establishment of the hierarchy structure}

The criteria and sub-criteria which characterizing the maintenance strategy for medical equipment are defined based on experts judgments of Tunisian Hospital and the literature on the criticality of medical equipment. Based on the literature, we defined seven main criteria and 6 sub-criteria for the selection maintenance strategy as follows:

- A: Degree of complexity of the maintenance: It depends on the maintenance requirements for medical equipment. In general, maintenance activity requires resources of three different types: labor, cost and tools. According to Fennigkoh and Smith (1989), there are three classes (grade): 1) Advanced mechanical, pneumatic or hydraulic equipment that requires extensive maintenance, 2) equipment with average maintenance requirements that just requires 
verification of performance and safety tests, and 3) equipment that receives only visual inspections and basic performance checks is classified with minimal maintenance requirements.

- B: Function: It is the main purpose for which the equipment is used (Taghipour et al. 2011). Four groups according to the criticality of the mission are classified by Fennigkoh and Smith (1989): Therapeutic, Diagnostic, Analytical, and Miscellaneous, and a grade from one to nine is allocated to this criterion. In our model, we used the same.

- C: Risk: it defines one of the most important criteria in selecting maintenance strategy. This criterion cannot be considered as a simple number allocated to an equipment. Then, the risk can be estimated as a function of four sub-criteria: detectability, frequency, safety and downtime.

- $\quad C_{1}$ : Detectability: This sub-criterion is the ability to detect a potential failure before it occurs.

- $\quad C_{2}$ : Frequency: The frequency of failures is the likelihood of a failure occurrence (Taghipour et al.2011).

- $\quad C_{3}$ : Safety: A potential failure of an equipment can result in injury, or even death of patients and users. This sub-criterion is considered very important to calculate the risk.

- $C_{4}$ :Downtime: Downtime is the average time that an equipment is not functional due to a potential failure.

These input data can be extracted or estimated from the history of maintenance work orders. We classified this criterion into 3 classes (grade): high, medium and low risk

- D: Degree of importance of the mission: This criterion is considered for calculating devices criticality and describes the degree to which a medical equipment is crucial to the health care delivery process of the hospital (Taghipour et al. 2011). It depends on two sub-criteria: utilization rate and availability of alternative devices. 
Quantitative techniques for medical equipment maintenance management

- $D_{1}$ : Utilization rate: The medical device utilization rate is calculated as the average hours of its usage per week dividing by the maximum number of hours per week. In our case, the maximum number of hours per week is considered to 48 hours per week.

- $D_{2}$ : Availability of alternative devices: According to Taghipour et al. (2011), the number of patients served per similar devices has an impact on the availability of these devices. The availability of alternative devices is considered as a function of the number of identical devices and their demand per unit time.

Three classes (grade) are considered for this criterion: necessary, important and critical.

- E: Age: The failure rate of a system or component usually depends on time and is calculated as the frequency with which the system or component fails (Khalaf et al. 2012). We can obtain the life span of a group of similar devices from Hospital History (from starting date of the equipment and date it reform) (Taghipour et al. 2011). We find that the average life span of medical equipment is 10 years. For this reason, two classes are considered to classify medical devices: new equipment which has $\leq 10$ years, old equipment otherwise.

- F: Recalls and user errors: The number of recalls and the number of fault alerts that may occur for an equipment due to user errors. This criterion should be deemed important in ranking medical devices for maintenance activities. US Food and Drug Administration (FDA) has classified this criterion into three classes according to the level of hazard involved (Taghipour et al. 2011).

- G: Classes of equipment: The classification of medical devices has the main purpose to assign a risk level and allocate necessary control and measurement to that level. The aim is to assure the safety and the effectiveness of medical devices. According to US Food and Drug Administration (FDA), Medical devices can be divided into 4 classes corresponding to the level of risk: class I: low degree of risk, 
class II a: medium degree of risk, class II b: high degree of risk, and class III: very high degree of risk.

To achieve our objective of selecting the appropriate maintenance strategy for medical equipment, we consider three maintenance strategies used in Tunisian Hospitals: Time-Based Maintenance, Condition-Based Maintenance, and Corrective Maintenance as alternatives in the AHP analysis. Then, we place all the required criteria for selecting the maintenance strategy at the appropriate hierarchical level. In Figure 2 we

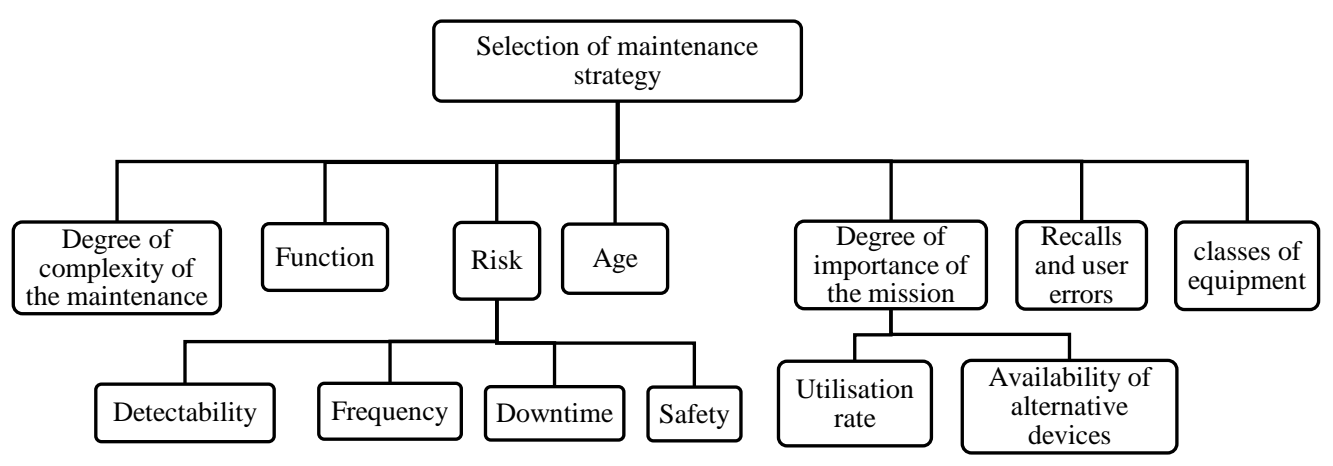

show our decision hierarchy used for the maintenance strategy selection.

Figure 2 An AHP Structure for selection maintenance strategy

\subsection{Establishment of comparative judgments}

Once all levels of the AHP hierarchy structure are defined as shown in Goal Fig.2, the next step is to make an interview with experts of the maintenance service (engineers and operators).A questionnaire is prepared for each experts of maintenance department to compare criteria and sub-criteria according to Saaty's scale. We concentrate on just two criteria of the same level (sub-criteria of corresponding criteria) Criterat the same time to determine weights using pairwise comparison. The preferences are quantified using integer values in interval of $[1,9]$ on

Sub-

$\underline{\underline{\text { criteria }}}$ 
Saaty's scale. The meaning of each value is explained in Table 1 (Shyjith et al. 2008) .

Table 1 AHP comparison scale (Saaty's scale)

\begin{tabular}{lc}
\hline \multicolumn{1}{c}{ Verbal judgments } & Degree of preferences \\
\hline Equally preferred & 1 \\
$\begin{array}{l}\text { Moderate importance and judgement } \\
\text { over another }\end{array}$ & 3 \\
Strong or essential favor of one over another & 5 \\
Very strong favor of one & 7 \\
Absolute favor of one over another & 9 \\
Intermidiate values between two adjacent judgments & $2,4,6,8$ \\
\hline
\end{tabular}

After developing all pairwise comparison matrices of the selection criteria, we calculate the concistency index (CI) using Eq. (2) where $n$ is the total number of selection criteria and $\lambda_{\max }$ is the maximum eigenvalue of the pairwise matrix (Shyjith et al. 2008).

$C I=\frac{\left(\lambda_{\max }-n\right)}{(n-1)}$

The consistency ratio $(C R)$ is calculated as a proportion of $C I$ as compared to a known random consistency index $(R I)$, see Eq. (3), (Shyjith et al. 2008):

$C R=\frac{C I}{R I}$

The relative importance weighting criteria and sub-criteria, $w_{i}$, obtained by AHP is presented in Table 2 and Table 3. 
The values given in Table. 2 and Table. 3 represent the reponsible of maintenance service opinion of a Tunisian Hospital (case of 'Habib Bourguiba Sfax' hospital). It is worthnoting a different group of experts from another hospital may give different results.

Table 2 Criteria weight

\begin{tabular}{ccccccccc}
\hline Criteria & $A$ & $B$ & $C$ & $D$ & $E$ & $F$ & $G$ & $\boldsymbol{W}_{\boldsymbol{i}}$ \\
\hline A & 0,079 & 0,084 & 0,048 & 0,036 & 0,302 & 0,147 & 0,191 & $\mathbf{0 , 1 3}$ \\
B & 0,393 & 0,419 & 0,570 & 0,215 & 0,302 & 0,235 & 0,255 & $\mathbf{0 , 3 4}$ \\
C & 0,314 & 0,140 & 0,190 & 0,358 & 0,181 & 0,206 & 0,191 & $\mathbf{0 , 2 3}$ \\
D & 0,157 & 0,140 & 0,038 & 0,072 & 0,015 & 0,147 & 0,255 & $\mathbf{0 , 1 2}$ \\
E & 0,016 & 0,060 & 0,063 & 0,287 & 0,060 & 0,088 & 0,032 & $\mathbf{0 , 0 9}$ \\
F & 0,016 & 0,052 & 0,027 & 0,014 & 0,020 & 0,029 & 0,013 & $\mathbf{0 , 0 2}$ \\
G & 0,026 & 0,105 & 0,063 & 0,018 & 0,121 & 0,147 & 0,064 & $\mathbf{0 , 0 8}$ \\
\hline
\end{tabular}


Quantitative techniques for medical equipment maintenance management

Table 3 Corresponding Criteria and sub-criteria weighting values and sub-criteria weighting values

\begin{tabular}{|c|c|c|c|c|c|}
\hline Criteria $i$ & $\begin{array}{c}\text { Grade } \\
\left(G_{i}\right)\end{array}$ & $\begin{array}{c}\text { Weights } \\
\quad\left(W_{i}\right)\end{array}$ & Sub-criteria ij & $\begin{array}{c}\text { Grade } \\
\left(G_{i j}\right)\end{array}$ & $\begin{array}{c}\text { Weights } \\
\left(W_{i j}\right)\end{array}$ \\
\hline $\begin{array}{l}\text { A : Degree of } \\
\text { complexity of } \\
\text { the maintenance }\end{array}$ & 3 & 0.13 & & & \\
\hline B : Function & 9 & 0.34 & & & \\
\hline \multirow[t]{5}{*}{ C: Risk } & & & $\begin{array}{l}C_{1}: \\
\text { Detectahility }\end{array}$ & 3 & 0.28 \\
\hline & 3 & 0.23 & & & \\
\hline & & & $C_{2}:$ Frequency & 3 & 0.48 \\
\hline & & & $C_{3}$ : Safety & 3 & 0.06 \\
\hline & & & $C_{3}$ : Downtime & 3 & 0.18 \\
\hline \multirow{2}{*}{$\begin{array}{l}\mathrm{D}: \text { Degree of } \\
\text { importance of } \\
\text { the mission }\end{array}$} & 3 & 0.12 & $\begin{array}{l}D_{1}: \text { Utilization } \\
\text { rate }\end{array}$ & 4 & 0.75 \\
\hline & & & $\begin{array}{l}D_{2} \text { : Availability } \\
\text { of alternative } \\
\text { devices }\end{array}$ & 2 & 0.25 \\
\hline E : Age & 2 & 0.09 & & & \\
\hline $\begin{array}{l}\mathrm{F}: \text { Recalls and } \\
\text { user errors }\end{array}$ & 3 & 0.02 & & & \\
\hline $\begin{array}{l}\text { G:Classes of } \\
\text { equipment }\end{array}$ & 4 & 0.08 & & & \\
\hline
\end{tabular}

Once all criteria and sub-criteria weights are calculated, we evaluated the expert' judgement by calculating the insconsistency ratio $(C R)$ using the maximum eigenvalue $\lambda_{\max }$ and the random consistency index $(R I)$ as shown 
is Eq.(2) and Eq.(3). Calculating the inconsistency ratio in pairwise comparaison of different criteria allows the model to be able to produce more precise and consistent criteria's weights compared to direct assignment of the weights. If $C R<0.1$ the results are acceptable and indicate a good level of consistency in the comparative judgements (Saaty 1994). Table 4 shows the consistancy results. We conclude that the calculated weights of criteria are consistant.

Table 4 Inconsistency ratio for pair wise comparison Matrix

$\begin{array}{cc}\text { Criteria } & \text { Result } \\ \lambda_{\max } & 7.567 \\ \text { Consistency index }(C I) & 0,094 \\ \text { Inconsistency ratio }(C R) & 0.072<0.1 \text { then we have a good level of consistency } \\ \text { in the comparative judgements }\end{array}$

We are ready to calculate the criticality of all medical equipment and to rank them. Each device should be evaluated under every covering criterion; the lowest level criterion or sub-criterion attached to the alternatives and assigned an appropriate grade (Saaty 2008). Then, the calculation of the criticality score of medical equipment can be obtained as the weighted sum of the criteria $\left(W_{i}\right)$ and their corresponding grade $\left(G_{i}\right)$ as shown in Eq.4:

Criticality score $=\sum_{i=1}^{7} W_{i} * G_{i}$

Only for criterion which has sub-criteria the grade $G_{i}$ depend on the weights and grade of sub-criteria as in Eq.5:

$G_{i}=\sum_{\forall j} W_{i j} * G_{i j}$

In our proposed model, medical equipment could have a criticality score between 1 and 5.12. The value 1 is obtained when we consider the lowest scores $\left(G_{i}\right)$ from all criteria and sub-criteria multiplying by its weights $\left(W_{i}\right)$. The total score 5.12 is for the medical equipment which gets the highest score $\left(G_{i}\right)$ of each single criterion $i$ and sub-criterion $j$ multiplying by corresponding weights. 
Quantitative techniques for medical equipment maintenance management

For example, for an ANESTHESIA VENTILATEUR type DRAGER JULIEN, the criticality score value is,

$(2 \times 0.13)+(9 \times 0.34)+[(1 \times 0.28+3 \times 0.48+3 \times 0.06+2 \times 0.18) \times 0.23]+$ $[(4 \times 0.75+1 \times 0.25) \times 0.12]+(2 \times 0.09)+(2 \times 0.02)+(3 \times 0.08)=4.69$.

Based on the weights of the criteria (sub-criteria) and the grade values assigned to each criterion and sub-criterion, the equipment criticality are determined and ranked according to an increasing order. In the following section, we shall classify the three maintenance strategies, called alternatives, according to their importance.

\subsection{Classification of maintenance strategies by TOPSIS}

TOPSIS (Technique for Order Performance by Similarity to Ideal Solution) is a useful technique in dealing with Multi-Criteria Decision Making (MCDM) problems in the real world (Hwang and Yoon 1981). It is based on the simple concept of choosing the best alternative having the shortest distance from the positive-ideal solution and the longest distance from the negative-ideal solution. The positive-ideal solution is the one that is regarded as the maximal benefit. In contrast, the negative ideal solution is the one that has the minimal benefit and the worst value of alternatives. These two ideal solutions are found according to experts' judgments. Finally, the alternatives are ranked with respect to the relative closeness to the ideal solution (Shyjith et al. 2008).

Based on values proposed by the responsible of the maintenance service, the performance matrices of corresponding alternatives (maintenance strategies) are constructed. Let us denote $a_{i j}, i=1, \ldots, n, j=1, \ldots, m$, the corresponding value in the performance matrices with $n$ is the total number of criteria and sub-criteria (in our case according to Table $3 n=9$ ), $m$ is the number of alternatives (in our case $m=3$ being corrective, time-based, and condition-based maintenance). We normalize the $a_{i j}$ as given in Eq. (6):

$a_{i j}^{\prime}=\frac{a_{i j}}{\sqrt{\sum_{j=1}^{j=m} a i j^{2}}} \quad ; i=1, \ldots, n ; j=1, . ., m$

The next step is to create the weighted normalized decision matrix (see Eq.7). For our case, this is obtained from the AHP method.

$x_{i j}=a_{i j}^{\prime} * W_{i}, i=1, \ldots, n, j=1, \ldots, m$. 
Where $W_{i}$ is the weight of criterion $i$, see Table 3 .

We consider $A_{i}^{+}$the positive-ideal solution, defined as the best performance score result of all alternatives on a criterion and $A_{i}^{-}$the negative-ideal solution defined as the worst performance score results of all alternatives on a criterion (Shyjith et al. 2008). Then, $A_{i}^{+}$and $A_{i}^{-}$for all criteria and sub-criteria are calculated as follows (Hwang and Yoon 1981):

$A_{i}^{+}=\operatorname{Max}\left(x_{i j}, j=1, \ldots, m\right), \quad i=1, \ldots, n ; j=1, \ldots, m$

$A_{i}^{-}=\operatorname{Min}\left(x_{i j}, j=1, \ldots, m\right), \quad i=1, \ldots, n ; j=1, \ldots, m$

Once the positive and negative ideal solutions are defined, we calculate the distance between the ideal solution $\left(\mathrm{Dj}^{+}\right)$and the negative idealsolution $\left(\mathrm{Dj}^{-}\right)$ for each alternative as following in Eq. (10) and Eq. (11) (Hwang and Yoon 1981):

$D_{j}^{+}=\sqrt{\sum_{i=1}^{n}\left(x_{i j}-A_{i}^{+}\right)}, \quad i=1, \ldots, n ; j=1, \ldots, m$

$D_{j}^{-}=\sqrt{\sum_{i=1}^{n}\left(x_{i j}-A_{i}^{-}\right)}, j=i=1, \ldots, n ; j=1, \ldots, m$

The final step in TOPSIS method is calculating the similarity degree or the relative closeness $\left(R_{j}\right)$ to the ideal-positive solution (or equivalently the farthest from the negative-ideal solution) for each alternative as given in Eq. (12) (Hwang and Yoon 1981).

$R_{j}=\frac{D_{j}^{-}}{D_{j}^{+}+D_{j}^{-}}, j=1, \ldots, m$

The alternative with the maximum value of relative closeness represents the best one. Others alternatives are ranked in descending order of their relative closeness.

The maintenance strategies considered in our experimental study as alternatives of our hierarchy structure are: Time-based maintenance (TBM), Condition-Based maintenance (CBM), and corrective maintenance (CM). Table 5 shows the global weighted normalized performances matrix of the 
alternatives $\left(x_{i j}\right)$. These values are calculated by multiplying the normalized performance matrix and criteria weights obtained from AHP method.

Table 5 Overall importance degrees of maintenance strategies $\left(x_{i j} ; i=1, \ldots, 11\right.$; $j=1,2,3)$

\begin{tabular}{llll}
\hline \multicolumn{1}{c}{ Alternatives } & TBM & CBM & $C M$ \\
\hline Degree of complexity of the maintenance & 0,100 & 0,071 & 0,043 \\
Function & 0,227 & 0.202 & 0.152 \\
Detectability & 0,197 & 0.173 & 0.099 \\
Frequency & 0.335 & 0.261 & 0.224 \\
Safety & 0.049 & 0.029 & 0.019 \\
Downtime & 0.138 & 0.092 & 0.069 \\
Utilization rate & 0.482 & 0.542 & 0.301 \\
Availability of alternative devices & 0.139 & 0.093 & 0.186 \\
Age & 0.045 & 0.063 & 0.045 \\
Recalls and user errors & 0.011 & 0.016 & 0.057 \\
Class of the equipment & 0.043 & 0.005 & 0.036 \\
\hline
\end{tabular}

After the establishment of the weighting performances matrix, we find the positive and negative ideal solution $\left(A_{i}^{+}\right.$and $\left.A_{i}^{+}\right)$. Then, we calculate the distances using Eq.10 and Eq.11 as shown in Table 6 and Table 7. 
Table 6 The positive and negative ideal solution to corresponding criteria and sub-criteria

\begin{tabular}{|c|c|c|c|c|c|c|c|c|c|c|c|}
\hline 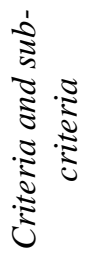 & 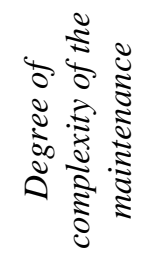 & 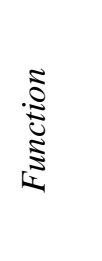 & 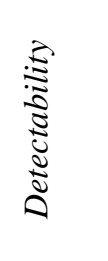 & 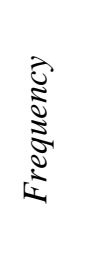 & $\begin{array}{l}\overrightarrow{3} \\
\frac{\omega}{5} \\
5\end{array}$ & 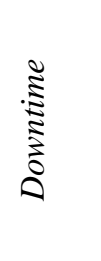 & 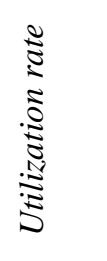 & 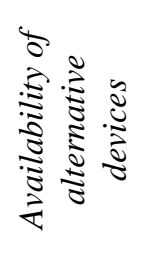 & $\stackrel{\infty}{\infty}$ & 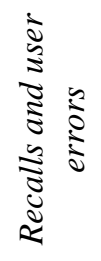 & 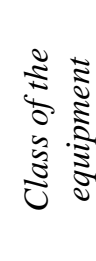 \\
\hline $\mathrm{A}_{\mathrm{i}}^{+}$ & 0,100 & 0,227 & 0,197 & 0,335 & 0,049 & 0,138 & 0,542 & 0,186 & 0,063 & 0,016 & 0,057 \\
\hline $\mathrm{A}_{\mathrm{i}}^{-}$ & 0,043 & 0,152 & 0,099 & 0,224 & 0,019 & 0,069 & 0,301 & 0,093 & 0,045 & 0,005 & 0,036 \\
\hline
\end{tabular}

Table 7 Calculating distance of each alternative to the ideal and negative solution

\begin{tabular}{cccc}
\hline & & & \\
Alternatives & TBM & $C B M$ & $C M$ \\
\hline$D_{j}^{+}$ & 0.08 & 0.137 & 0.308 \\
$D_{j}^{-}$ & 0.268 & 0.264 & 0.093 \\
\hline
\end{tabular}

The ranking of alternatives is obtained according to their relative closeness to ideal solution calculated via Eq. (12). Next, we obtained the ranking of maintenance strategies as shown in Figure 3.

Figure 3 Ranking Maintenance strategies according to their relative closeness

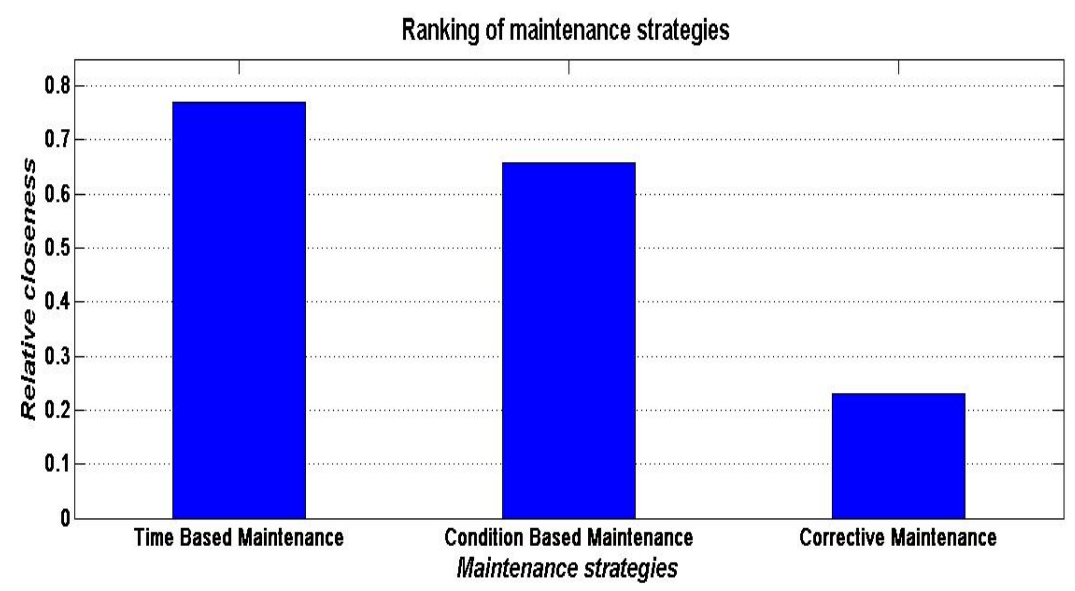


Our results show that TBM with relative closeness $R_{T B M}=0.771$ is the best strategy for the highly critical equipment, then CBM with $R_{C B M}=0.659$, and last $\mathrm{CM}$ with corresponding relative closeness $R_{C M}=0.231$.

In addition to all criteria considered previously, the repair cost (labor, tools and spare parts) is integrated as a sub-criterion of the risk by Taghipour et al.(2011). They consider according to experts' point of view that CBM is the best strategy for critical medical equipment, then TBM, and last CM. Likewise, maintenance costs that contains fixed costs (e.g costs of spare parts) and other variable costs as the cost of maintenance experts is considered by Jamshidi et al. (2015). Then, the classification of maintenance strategies is: $\mathrm{CBM}$ with high priority for critical devices, then TBM and CM.

To verify this ranking, we applied our approach AHP coupled with TOPSIS technique for the same criteria added to the cost. Table. 8 shows the new relative importance weighting criteria and sub-criteria obtained by AHP method. 
Z.Ben Houria, M.Masmoudi, A.Al Hanbali, I.Khatrouch and F.Masmoudi

Table 8 Corresponding Criteria and sub-criteria weighting values (with cost sub-criteria)

\begin{tabular}{|c|c|c|c|}
\hline Criteria & $W_{i}$ & Sub-criteria & $W_{i}$ \\
\hline $\begin{array}{l}\text { A: Degree of complexity } \\
\text { of the maintenance }\end{array}$ & 0.13 & & \\
\hline B: Function & 0.34 & & \\
\hline \multirow[t]{5}{*}{ C: Risk } & 0.23 & $C_{1}:$ Detectability & 0.17 \\
\hline & & $C_{2}:$ Frequency & 0.26 \\
\hline & & $C_{3}:$ Safety & 0.05 \\
\hline & & $C_{4}:$ Downtime & 0.13 \\
\hline & & $C_{5}:$ Cost & 0.40 \\
\hline \multirow{2}{*}{$\begin{array}{l}\text { D: Degree of importance } \\
\text { of the mission }\end{array}$} & 0.12 & $D_{1}:$ Utilisation rate & 0.75 \\
\hline & & $\begin{array}{l}D_{2}: \text { Availability of } \\
\text { alternative devices }\end{array}$ & 0.25 \\
\hline E: Age & 0.09 & & \\
\hline F: Recalls and user errors & 0.02 & & \\
\hline $\begin{array}{l}\text { G: Class of the } \\
\text { equipment }\end{array}$ & 0.08 & & \\
\hline
\end{tabular}

Once the relative importance weighting are defined, we calculate the relative closeness of each maintenance strategy and rank the alternatives as presented in Figure 4. 
Figure 4 New ranking Maintenance strategies (with cost sub-criterion) according to their relative closeness

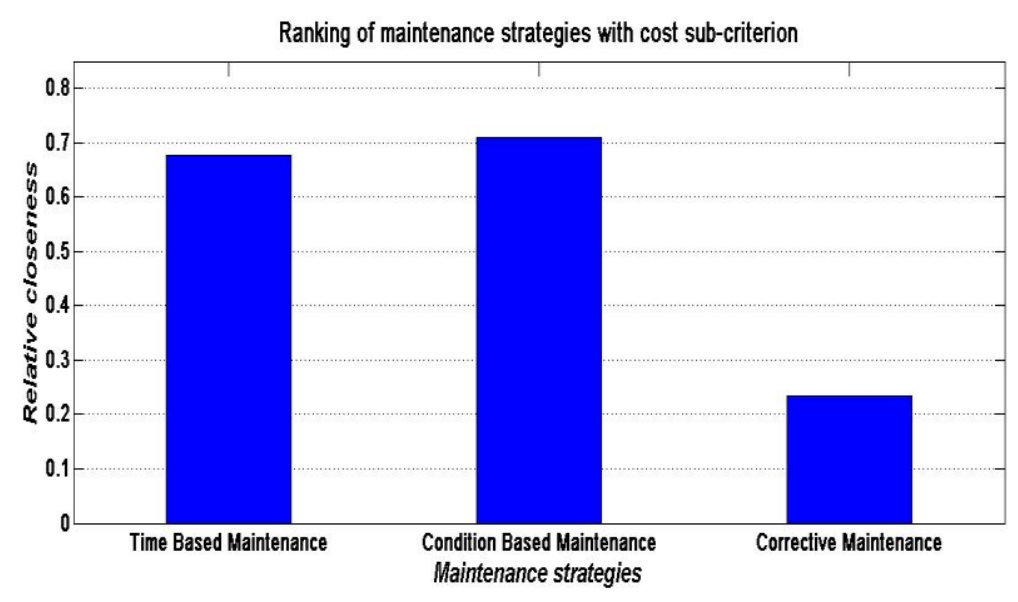

Results showed that the ranking of maintenance strategies is as follows:

- Ranking1: 1-Time-based maintenance (TBM), 2-Condition-Based maintenance (CBM) and 3-Corrective maintenance, when maintenance cost is not considered in the device criticality study (Fig. 3).

- Ranking2: 1-Condition-based maintenance (CBM), 2-Time-Based maintenance (TBM) and 3-Corrective maintenance (Taghipour et al. 2011; Jamshidi et al. 2015), when maintenance cost is considered in the device criticality study (Fig. 4).

Thus, our approach of AHP coupled with TOPSIS technique confirms scientifically these two different ranking depended on cost criterion. According to the responsible of the maintenance service, the Ranking1 is more suitable. Moreover, the cost factor will be included as a constraint in the MILP formulation. So including this factor in the weight calculation will overestimate its impact on the maintenance strategies score.

\subsection{MILP for maintenance strategy selection}

Now given that the equipment criticality was found and maintenance strategies were classified, we are ready to determine which subset of equipment will be maintained according to TBM, CBM, and CM. In order 
to do this, we define two criticality thresholds $T_{1}$ and $T_{2}$ separating the three possible strategies to determine the maintenance strategy for each device (See Fig. 5). Equipment with criticality scores smaller than $T_{1}$ follow CM strategy, then between $T_{1}$ and $T_{2}$ are associated to CBM strategy, and larger than $T_{2}$ follow TBM strategy.

Figure 5 Devices and corresponding maintenance strategy according to thresholds $T_{1}$ and $T_{2}$

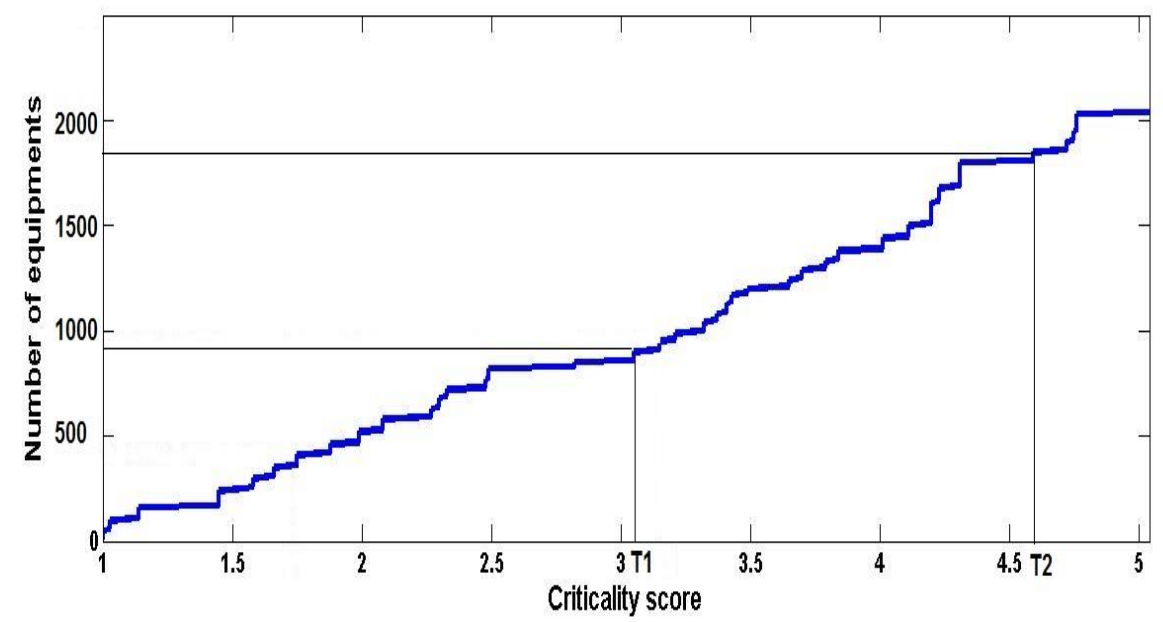

The equipment maintenance cost is given according to the corresponding maintenance strategy. Thus, the aim of this section consists of defining the best values of $T_{1}$ and $T_{2}$ which allow selecting the adequate strategy per equipment and maximizing the preventive maintenance activities. The higher the threshold $T_{1}$ is, the less number of equipment are assumed to undergo preventive maintenance and vice-versa.

To solve the problem, we propose in the following an efficient MILP model to minimize $T_{1}$ and $T_{2}$ values (e.g. maximize preventive maintenance activity) with respecting the budget allocated to maintenance service. As an objective function, we considered a weighted sum of $T_{1}$ and $T_{2}$ which are given by TOPSIS. In our model, an estimation of the maintenance cost per maintenance strategy per equipment and reserved budget are considered as given input data.

The indexes, parameters and decision variables, which are considered in our model, are as following:

\section{Indexes:}


$i$ : index for number of devices in total $(n) ; i=1, \ldots, n$.

$j$ : index for maintenance strategies; $j=1 . .3$. As given that possible maintenance strategies are: corrective (1), condition-based (2) and timebased (3) respectively.

\section{Parameters:}

$C r_{i}$ : Criticality of equipment $i$, the equipment are ranked according to an increasing order of criticality $\left(C r_{i} \leq C r_{i+1}, \forall i=1, \ldots, n-1\right)$.

$\operatorname{Cost}_{i, j}$ : Maintenance cost of each device $i$ according to the maintenance strategy $j$.

Budget: the amount of money for maintenance service.

\section{Decision variables:}

$T_{1}$ : Limit of criticality between corrective maintenance $(j=l)$ and Condition-based $(j=2)$

$T_{2}$ : Limit of criticality between Condition-based $(j=2)$ and Time-based $(j=3)$

$X_{i, j}$ : Boolean variable, equal to 1 if maintenance strategy $j$ is considered for device $i, O$ otherwise.

The proposed MILP model is the following:

Maximize $\quad 0.23 * \sum_{i=1}^{i=n} X_{i, 1}+0.66 * \sum_{i=1}^{i=n} X_{i, 2}+0.77 * \sum_{i=1}^{i=n} X_{i, 3}$

\section{Subject to:}

$$
\begin{aligned}
& \sum_{i=1}^{i=n} \sum_{j=1}^{j=3} \operatorname{Cost}_{i, j} * X_{i, j} \leq \text { Budget } \\
& \sum_{j=1}^{j=3} X_{i, j}=1 \forall i=1, \ldots, n \\
& X_{i, 1}+2 X_{i, 2}+4 X_{i, 3} \leq X_{i+1,1}+2 X_{i+1,2}+4 X_{i+1,3} \\
& \forall i=1, \ldots, n-1 \\
& \sum_{i=1}^{i=n-1} \mathrm{Cr}_{i} *\left(X_{i, 1}-X_{i+1,1}\right)=T_{1} \\
& \sum_{i=1}^{i=n-1} C r_{i} *\left(X_{i, 1}-X_{i+1,1}+X_{i, 2}-X_{i+1,2}\right)=T_{2}
\end{aligned}
$$


$1 \leq T_{1}<T_{2} \leq 5.12$

$X_{i, j} \in\{0,1\} \quad \forall i=1, \ldots, n, \forall j=1 \ldots 3$

In relation (13), the objective function is to maximize the weighted (taking the relative closeness results of TOPSIS as a strategy weight) sum of devices to be maintained within CM, CBM and TBM strategies. Since, the weights of CBM and TBM are higher than the CM weight the optimization problem will yield to the most possible number of equipment (satisfying the constraints) to receive a CBM or a TBM maintenance. Constraint (14) ensures the total cost of maintenance is smaller than the available Budget. Constraint (15) guarantees the assignment of only one maintenance strategy per device. As devices are ordered respecting the increasing score of the criticality $\left(\mathrm{Cr}_{i}\right)$, constraint (16) ensures that, for two devices ' $\mathrm{v}$ ' and ' $\mathrm{s}$ ' in $\{1, \ldots, n\}$, if $v \leq s$ (i.e. $C r_{v} \leq C r_{s}$ ), then the corresponding strategies $S_{v}$ and $S_{s}$ in $\{1,2,3\}$ are $S_{v} \leq S_{s}$. For example, if the strategy maintenance for device 's 'is Time-based, then all equipment placed after ' $\mathrm{s}$ ' are selected for Time-based maintenance. Constraint (17) defines the criticality threshold $T_{1}$ between the devices under the corrective maintenance strategy and those under condition based maintenance strategies. $T_{1}$ represents the highest criticality of medical devices with corrective maintenance strategy, i.e., the criticality of device $i$ with $X_{i, 1}=1$ and $X_{i+1,1}=0$. Similarly, constraint (18) defines the threshold $T_{2}$ between condition and time based maintenance strategies. $T_{2}$ represents the highest criticality of medical devices with condition based maintenance strategy, i.e., the criticality of device $i$ with $X_{i, 2}=1$ and $X_{i+1,2}=0$. Constraint (19) specifies the domain of $T_{1}, T_{2}$ according to the minimal and the maximal criticality values defined in Section 3.1. This constraint ensures that $T_{1}$ is always less than $T_{2}$. Finally, constraint (20) specifies the domain of the decision variable $X_{i, j}$.

\section{Experimental results and data analysis}

The MILP model is implemented in "Cplex 12.5" and used a computer with a processor of the following characteristics Intel (R) Pentium (R) CPU 2020M 2.60 GHz. We consider real input data of the medical devices based on the 'Habib Bourguiba' hospital in Tunisia. We have tested our model with many instances varying between 20 and 2000 devices. In Table 9, we 
show the computation time in seconds to each of these instances. The largest case can be solved in a very short time of only $15.18 \mathrm{~s}$.

Table 9 Computation time for 8 different instances

\begin{tabular}{ccc}
\hline$N^{\circ}$ of instance & Number of devices & Computation time (seconds) \\
\hline 1 & 20 & 0.39 \\
2 & 50 & 0.56 \\
3 & 100 & 1.12 \\
4 & 200 & 1.52 \\
5 & 500 & 3.82 \\
6 & 1000 & 5.15 \\
7 & 1500 & 10.11 \\
8 & 2000 & 15.18 \\
\hline
\end{tabular}

For the case of 'Habib Bourguiba' Hospital, we have considered all devices (2000 medical devices). In fact, if we consider only a group of devices, the maintenance managers may allocate all the available budget to this group and the result will be to apply preventive maintenance activities for all of them which is not necessarily required. Criticality scores vary between 1 and 5.12 and classified in the increasing order. The main objective of this instance is to find the best values of the thresholds $\mathrm{T}_{1}$ and $\mathrm{T}_{2}$ to maximize the preventive maintenance activities with respect to the available budget. The computation is achieved in only 15.18 seconds. The results for selecting the maintenance strategy per device are summarized in Table 10. In this table, we find that all devices with criticality smaller than $3.04\left(=\mathrm{T}_{1}\right)$ should be maintained according to the CM strategy. However, those with criticality larger or equal than $4.66\left(=\mathrm{T}_{2}\right)$ should be maintained according to the TBM strategy. The remaining devices should be maintained according to CBM strategy. The available budget is set at 4,000,000TND (Tunisian Dinar) for all maintenance activities (corrective and preventive). The cost break down and the allocation of the devices between the different strategies are as follows: the CM strategy has 1219 (60.95\% of devices) allocated devices with a maintenance cost of $617,012 \mathrm{TND}$; the CBM strategy has 698 (34.9\% of devices) allocated devices with a maintenance cost of 2,842,336TND; Finally, the TBM has 83 (4.15\% of devices) allocated devices with a maintenance cost of 540,648TND. The deviation between the available budget and the total maintenance cost is around 4TND. 
Z.Ben Houria, M.Masmoudi, A.Al Hanbali, I.Khatrouch and F.Masmoudi

Table 10 MILP results for 2000 medical devices using CPLEX solver

\begin{tabular}{|c|c|c|c|c|}
\hline$N^{\circ}$ & Criticality & $\begin{array}{c}\text { Corrective } \\
\text { maintenance }\end{array}$ & $\begin{array}{c}\text { Condition based } \\
\text { maintenance }\end{array}$ & $\begin{array}{c}\text { Time } \\
\text { Based maintenance }\end{array}$ \\
\hline 1 & 1 & 1 & 0 & 0 \\
\hline . & . & . & . & . \\
\hline . & . & . & . & . \\
\hline . & . & . & . & . \\
\hline 1219 & 3.04 & 1 & $\mathbf{0}$ & $\mathbf{0}$ \\
\hline 1220 & 3.08 & 0 & 1 & 0 \\
\hline 1221 & 3.08 & 0 & 1 & 0 \\
\hline . & . & . & . & . \\
\hline . & . & . & . & . \\
\hline . & . & . & . & . \\
\hline 1916 & 4.66 & 0 & 1 & 0 \\
\hline 1917 & 4.66 & 0 & 1 & 0 \\
\hline 1918 & 4.69 & 0 & 0 & 1 \\
\hline 1919 & 4.69 & 0 & 0 & 1 \\
\hline$\cdot$ & · & . & . & . \\
\hline . & . & . & . & . \\
\hline 2000 & 5.12 & 0 & 0 & 1 \\
\hline
\end{tabular}

Hence, the computational study proves the effectiveness of our method in selecting the best maintenance strategy of each medical device according to its criticality score and the available budget. Added to that, our MILP model seeks to find the best number of equipment maintained with CBM and TBM to maximize the equipment availability and increase their reliability. Then, the different type of medical devices are considered to decide how the available budget should be allocated for the management of devices and which maintenance strategy should be applied for each device.

A sensitivity analysis is done to see the impact of varying the available maintenance budget on the set of devices, see Table 11. The analysis shows that when the maintenance budget (input) decreased the thresholds $\mathrm{T}_{1}$ and $\mathrm{T}_{2}$ (outputs) change in such way more equipment are maintenance according CM. The result also implies that the more the available budget is important, the more we have medical devices maintained within CBM and TBM and less devices with $\mathrm{CM}\left(\mathrm{T}_{1}\right.$ and $\mathrm{T}_{2}$ are decreased $)$ and vice versa.

Table 11 Results of varying the available maintenance budget

\begin{tabular}{cccccc}
\hline $\begin{array}{c}\text { \% of varying } \\
\text { factor }\end{array}$ & $T_{1}$ & $T_{2}$ & $\begin{array}{c}\text { \% of devices under } \\
\text { CM }\end{array}$ & $\begin{array}{c}\text { \% of devices } \\
\text { under CBM }\end{array}$ & $\begin{array}{c}\text { \% of devices } \\
\text { under TBM }\end{array}$ \\
\hline $\mathbf{- 1 5 \%}$ & 4.59 & 5.09 & $92.5 \%$ & $6.2 \%$ & $1.3 \%$ \\
$\mathbf{- 1 0 \%}$ & 4.26 & 5.04 & $84.6 \%$ & $11.95 \%$ & $3.45 \%$
\end{tabular}


Quantitative techniques for medical equipment maintenance management

\begin{tabular}{cccccc}
$\mathbf{- 5 \%}$ & 3.86 & 5.02 & $76.25 \%$ & $19.75 \%$ & $4 \%$ \\
$\begin{array}{c}\text { The initial } \\
\text { solution }\end{array}$ & $\mathbf{3 . 0 4}$ & $\mathbf{4 . 6 6}$ & $\mathbf{6 0 . 9 5 \%}$ & $\mathbf{3 4 . 9 \%}$ & $\mathbf{4 . 1 5 \%}$ \\
$\mathbf{+ 5 \%}$ & 2.63 & 4.66 & $52.4 \%$ & $42.5 \%$ & $5 \%$ \\
$\mathbf{+ 1 0 \%}$ & 1.56 & 4.63 & $41.6 \%$ & $52.65 \%$ & $5.75 \%$ \\
$\mathbf{+ 1 5 \%}$ & 1 & 4.46 & $0.05 \%$ & $89.5 \%$ & $10.45 \%$ \\
$\mathbf{+ 2 0 \%}$ & 1 & 3.78 & $0.05 \%$ & $70.3 \%$ & $29.65 \%$ \\
$\mathbf{+ 2 5 \%}$ & 1 & 2.42 & $0.05 \%$ & $51.25 \%$ & $48.7 \%$ \\
\hline
\end{tabular}

\section{Conclusions and perspectives}

In this paper, we use an integrated approach that includes several tools from the literature. The two main contributions presented in this paper are: (1) proposing an innovative Multi-criteria Decision Making (MCDM) methodology that combines the Analytical Hierarchy Process (AHP) and TOPSIS technique. AHP is used to calculate the criticality score of each device based on a set of selected criteria and sub-criteria. TOPSIS technique is used to rank the considered maintenance strategies, and (2) development of a MILP model to assign the adequate maintenance strategy to each medical device while respecting the available budget. The results of the proposed framework are validated with a real data set collected from a Tunisian Hospital.

Our proposed model is original and innovative. It produces an efficient prioritization of medical devices and not a simple ranking. Furthermore, it allows managers to select the best maintenance strategy for medical device according to their criticality scores. This framework can be implemented as a module into the software for biomedical maintenance of the hospital and ensures high availability for high critical medical devices. Moreover, it is relevant to any other hospital from other countries by modifying data according to corresponding maintenance history.

In our proposed model, the pairwise comparison matrices are constructed using Saaty's scale. Fuzzy logic can be used to minimize subjective experts' judgments.

In future works, we will develop a mathematical model including internalization/externalization and contract selection problem in order to increase the availability of medical devices and optimize the maintenance activity and budget allocation to maintenance tasks. 


\section{References}

Almeida, A.T. and Bohoris, G.A. (1995), "Decision theory in maintenance decision making”, Journal of Quality in Maintenance Engineering, Vol. 1 No. 1, pp. 39-45.

Association for the Advancement of Medical Instrumentation (AAMI,1985) 'Guidelines for the Establishing and Administering Medical Instrumentation Maintenance Programs'. USA.

Bevilacqua, M. and Braglia, M. (2000), "The analytic hierarchy process applied to maintenance strategy selection", Reliability Engineering and System Safety, Vol. 70 No. 1, pp. 71-83.

Ding S.-H., Kamaruddin S., Abdul Azid I. (2014), "Development of a model for optimal maintenance policy selection", European J. of Industrial Engineering, Vol. 8, No.1, pp. 50-68.

Ebeling, C. (2004), An introduction to reliability and maintainability engineering. Tata McGraw-Hill Education, 2004.

Ertugrul, I. and Karakasoglu, N. (2007), "Comparison of fuzzy AHP and fuzzy TOPSIS methods for facility location selection", International Journal of Advanced Manufacturing Technology, pp. 783-95.

Fennigkoh L.and Smith B. (1989). “Clinical equipment management”. Jcaho Ptsm Ser 2:514.

Food and Drug Administration, Protecting and promoting your Health, http://www.fda.gov.

Ghosh D., Roy S. (2010), “A decision-making framework for process plant maintenance”, European J. of Industrial Engineering, Vol. 4, No.1, pp. 78-98.

Hummel J., (2001). "Supporting medical technology development with the analytic hierarchy process", Wageningen:Grafischbedrijf Ponsen\& Looijenbv. p.199.

Hwang, C.L. and Yoon, K. (1981), Multiple Attribute Decision Making Methods and Applications, Springer, New York, NY.

Ilangkumaran M. and Kumanan S. (2009), "Selection of maintenance policy for textile industry using hybrid multi-criteria decision making approach", Journal of Manufacturing Technology Management, Vol. 20 No. 7, 2009 pp.1009-1022.

Ivlev I., Kneppo P. and Bartak M. (2014), 'Multicriteria decision decision analysis: a multifaceted approach to medical equipment management', Technological and Economic Development of Economy, Volume 20, Issue 3, 2014

Jamshidi A., Abbasgholizadeh Rahimi S., Ait-kadi D. and Ruiz A. (2014), "Medical devices Inspection and Maintenance; a Literature Review", Proceeding of the 2014 Industrial and Systems Engineering Research Conference.

Jamshidi A, Abbasgholizadef Rahimi S., Ait-kadi D., Ruiz A., (2015) A comprehensive fuzzy risk-based maintenance framework for prioritization of medical devices,Applied Soft Computing 32 (2015) 322-334.

Khalaf A.B., Hamam Y., Djouani K. (2012) 'Mathematical Maintenance Model for Medical Equipment', University of Versailles Saint -Quentin-en- Yvelines. 